16 | 2021

Simone et André Schwarz-Bart : nouvelles approches de l'oeuvre

\title{
Pluies et vents sur Solitude
}

Étude de la réception des œuvres d'André et Simone Schwarz-Bart en Guadeloupe en 1972

\section{Fanny Margras}

\section{(2) OpenEdition}

Journals

Édition électronique

URL : https://journals.openedition.org/coma/6625

DOI : $10.4000 /$ coma.6625

ISSN : 2275-1742

Éditeur

Institut des textes \& manuscrits modernes (ITEM)

Référence électronique

Fanny Margras, «Pluies et vents sur Solitude », Continents manuscrits [En ligne], 16 | 2021, mis en ligne le 15 avril 2021, consulté le 11 janvier 2023. URL : http://journals.openedition.org/coma/6625 ; DOI : https://doi.org/10.4000/coma.6625

Ce document a été généré automatiquement le 11 janvier 2023.

\section{(c) (i) () $\Theta$}

Creative Commons - Attribution - Pas d'Utilisation Commerciale - Pas de Modification 4.0 International - CC BY-NC-ND 4.0

https://creativecommons.org/licenses/by-nc-nd/4.0/ 


\title{
Pluies et vents sur Solitude
}

\author{
Étude de la réception des œuvres d'André et Simone Schwarz-Bart en \\ Guadeloupe en 1972
}

Fanny Margras

1 Lorsqu'en 2015 paraît L'Ancêtre en Solitude ${ }^{1}$, co-signé par André et Simone Schwarz-Bart et publié après la mort d'André Schwarz-Bart, Jérôme Garcin rappelle la réception du troisième roman de l'auteur, La Mulâtresse Solitude ${ }^{2}$ :

Mais le livre, qui illustrait l'idée de métissage, la symbiose des civilisations, fut mal accueilli. « Lorsqu'on est précurseur, dit aujourd'hui Simone [Schwarz-Bart], on le paie cher. Les Antillais ne voulaient pas alors de leur histoire. » On fit, à son auteur, un procès en légitimité - que pouvait-il savoir, lui, l'enfant de Metz d'origine polonaise, l'ancien résistant, l'homme blanc, de la geste caribéenne ? - et on le soupçonna même de s'être approprié l'œuvre de sa femme³.

Poussant d'un cran les affirmations de Yann Plougastel, qui évoquait «l'incompréhension, voire l'hostilité suscitée par ce récit d'un épisode de l'histoire antillaise ${ }^{4}$ ", et reprenant à son compte les propos introductifs de Simone Schwarz-Bart au roman, J. Garcin semble certain que le mystérieux silence d'André Schwarz-Bart puise ses origines dans l'accueil désastreux que réserva la communauté antillaise à son roman sur l'esclavage. Si l'un comme l'autre emploient des formules impersonnelles, le pronom "on » dominant et les tournures passives au complément d'agent évasif ou inexistant régissant la plus grande partie des phrases, sous couvert, donc, de la prétérition, la thèse est cependant clairement formulée. Pourtant, rien ne semble attester l'accueil glacé du roman en Guadeloupe dans les années 1970. En dépit de nos recherches dans les archives de presse de la période 1972-1973, nous n'avons retrouvé les traces d'aucune attaque immédiate reprochant au «Blanc » d'avoir « osé écrire sur les Antilles ", ce qui permettrait d'expliquer le long et mystérieux silence littéraire d'André Schwarz-Bart, de 1972 jusqu'à sa mort, silence uniquement troublé, en 1988-1989, par la publication de l'encyclopédie Hommage à la femme noire ${ }^{5}$, à la rédaction duquel il collabore officiellement.

3 Si certaines critiques virulentes ont pu être émises contre l'écrivain, il semble qu'elles émanent d'une très petite frange d'intellectuels, qui n'appartiennent pas tous à la communauté antillaise francophone, qu'elles se développent bien après les 
années 1970, et que la question de la légitimité de l'écrivain ne se pose pas véritablement en termes de couleur de peau. Nous montrerons l'absence d'une réaction à vif qui aurait suscité un débat racialiste responsable du silence d'André Schwarz-Bart. Nous focalisant sur les premiers mots écrits par des journalistes et critiques lors de la parution des œuvres "antillaises", nous nous attarderons donc ici sur la réception immédiate de La Mulâtresse Solitude et de Pluie et Vent sur Télumée Miracle 6 au début des années 1970 en Guadeloupe. Comment les créations des deux écrivains ont-elles été immédiatement perçues par ceux qui les ont lues et qui ont peut-être découvert à travers elles une autre manière d'écrire, de penser et de se penser? C'est ce que nous nous proposons d'explorer en amorçant une réflexion sur ledit " procès en légitimité7 " qui aurait été intenté à André Schwarz-Bart, entraînant dans son sillage le silence de l'écrivain.

\section{Lire les Schwarz-Bart dans la Guadeloupe des années 1970}

Dans la période mouvementée qui suit immédiatement les troubles politiques de la fin des années 1960 dans les Antilles françaises, notamment le «massacre » de mai $1967^{8}$, peu de romans contemporains trouvent un écho au sein de la presse antillaise. Toutefois, en dépit de ce contexte, quelques mentions et critiques, en Guadeloupe, dès la parution d'Un plat de porc aux bananes vertes au Seuil en 1967, montrent un intérêt pour les romans schwarzbartiens9.

5 Nous avons fait le choix de travailler sur un large corpus de périodiques conservés aux Archives départementales de la Guadeloupe ${ }^{10}$. Il s'agissait d'étudier l'accueil réservé en Guadeloupe aux œuvres d'André et Simone Schwarz-Bart, par des critiques et journalistes, spécialistes ou néophytes du monde littéraire. Outre la recension de l'écrivaine guadeloupéenne Maryse Condé dans Présence Africaine, nous avons donc systématiquement parcouru tous les périodiques locaux de janvier 1972 à février 1973 pouvant accueillir une note de lecture relative à la parution de La Mulâtresse Solitude et de Pluie et Vent sur Télumée Miracle, respectivement en février et octobre 1972.

\section{La Mulâtresse Solitude (1972) : un bruissement de feuilles dans un désert médiatique}

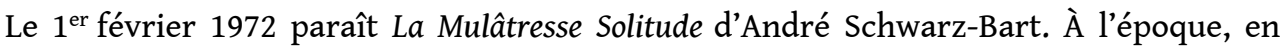
Guadeloupe, on recense peu de publications s'affichant comme des revues littéraires : en effet, en 1971, la revue Parallèles, éditée à Paris, distribuée (entre autres) en Guadeloupe, et s'attachant à valoriser le patrimoine littéraire et culturel antillais ${ }^{11}$, cesse de paraître. La revue $A c o m a^{12}$, elle aussi éditée à Paris, se focalise sur la Martinique, et interroge l'ancrage culturel de cette île au cœur des problématiques politiques qui lui sont contemporaines. Peu à peu, la plupart des journaux, préoccupés par un climat social tendu, se détourne du fait culturel et littéraire. Ainsi, dans les années 1972-1973, nous pouvons observer que la littérature s'efface de la presse, et ce d'autant plus si elle n'est pas ostensiblement militante et engagée contre la départementalisation, ou favorable à l'indépendance des colonies et de la Guadeloupe : devenu « nouvel espace d'investigation et de contestation ${ }^{13}$ », ce « support dogmatique 
de l'enfermement des choses et de la parole » est dénoncé, notamment par la revue CARE dans l'éditorial de son premier numéro ${ }^{14}$.

7 Dans ce contexte, on retrouve très peu d'articles dédiés aux romans ou à la fiction. Loin de l'explosion médiatique parisienne dont a fait l'objet Le Dernier des Justes en $1959^{15}$, le nouveau roman d'André Schwarz-Bart, La Mulâtresse Solitude, reste, à sa parution, et tout au long de l'année 1972, dans l'ombre des revendications sociales et politiques en Guadeloupe ${ }^{16}$.

En dehors du monde universitaire, seules deux revues à tirage réduit mentionnent ce roman. D'abord, en mai 1972, dans le mensuel culturel Guadeloupe 2000, fondé en 1970 par Édouard Boulogne et édité à Pointe-à-Pitre. Au contenu de ce mensuel, ayant pour objectif affiché de défendre la "Guadeloupe française", est ajoutée en 1972 une rubrique «Les Arts et les Lettres", puis une section «Lu pour vous... Sélectionné ce mois-ci à la vitrine des LIBRAIRIES ANTILLAISES », dans laquelle figure le 15 mai 1972, parmi une sélection d'études socio-culturelles, une note critique du roman d'André Schwarz-Bart :

De mère africaine - arrachée à son village de brousse par les trafiquants d'esclaves et de père inconnu - quelque marin du bateau négrier voguant vers la Guadeloupe -, elle n'est ni noire ni blanche, et même ses deux yeux sont de nuance différente. Enfant, on la surnommera «Deux-âmes ». Et finalement c'est sous le nom de «Solitude » qu'elle vivra à la Guadeloupe, dans les familles de Blancs qui l'ont achetée puis parmi les troupes de Noirs révoltés qu'elle rejoindra à grand peine dans leurs refuges des forêts de la Soufrière.

L'histoire se passe d'environ 1760 à 1802. L'abolition de l'esclavage décrétée par la Convention n'aura duré que le temps d'un rêve. Et Solitude, près de l'Africain Maïmouni qu'elle a découvert dans la forêt et dont elle partage la vie, a senti en elle-même "battre un cœur de négresse ». C'est elle, enceinte et soutenue par ses compagnons, qui anime le dernier combat. Capturée, elle est pendue après avoir donné naissance à son enfant.

Tel fut le destin tragique d'un personnage bien réel, sous les couleurs de la légende. L'auteur $d u$ Dernier des Justes en a rendu perceptibles «le bruit et la fureur " à travers la musique et la tendresse.

Ce livre est le premier d'un cycle qui se déroule de 1760 à nos jours. Le volume précédent, Un plat de porc aux bananes vertes publié sous la signature d'André et Simone SchwarzBart, en constituait le prélude.

9 La notice s'attache, dans un premier temps, à résumer le roman, en insistant sur l'engagement politique du personnage de Solitude. L'auteur n'apparaît qu'ensuite, au cœur d'un passage directement recopié de la quatrième de couverture de la première édition de La Mulâtresse Solitude au Seuil et que nous avons choisi de présenter ici en italiques. L'action politique semble être le véritable enjeu du roman; ce dernier ne paraît plus tout à fait appartenir à la fiction, ne serait-ce que par la sélection des études ${ }^{17}$ qui figurent dans la même section que lui.

La seconde mention de La Mulâtresse Solitude apparaît dans le bimensuel Église de Guadeloupe $^{18}$ qui se présente comme le "bulletin religieux du diocèse », compilant actualités sur la vie de l'église locale et du diocèse et articles de réflexion sur la Guadeloupe. On y retrouve quelques critiques de films, généralement une par numéro, de 1967 à 1972, mais une seule critique de roman, celle de La Mulâtresse Solitude par Rose-Marie Wendling en septembre 1972 :

Avec ce livre, André Schwarz-Bart poursuit la croisade contre le racisme qu'il avait entreprise dans Le Dernier des Justes.

Nous sommes aux Antilles, au milieu des Noirs arrachés à leur terre africaine par les 
marchands d'esclave au XVIII ${ }^{\mathrm{e}}$ siècle. Triste vie que la leur: elle est évoquée de manière tour à tour poétique et atroce.

Sur ce fond de tableau, une femme, la mulâtresse Solitude, se détache comme une forme irréelle, un être hors de la vie des humains, symbole de ces peuples transplantés. Pourtant, en cette esclave si humble et si docile, se cachent une âme passionnée et une force étonnante, qui feront d'elle le ferment de la révolution marronne.

D'autres écrivains se sont penchés sur ce problème des esclaves noirs d'Amérique. Leurs écrits ont suscité parfois l'horreur ou le dégoût. Rien de semblable ici. Il émane seulement de cette histoire une grande et douce tristesse. À aucun moment Schwartz-Bart (sic) ne porte de jugement; et, pour évoquer les faits les plus cruels, il se contente le plus souvent de demi-teintes. Respect pour les souffrances et les humiliations d'un peuple? Une chose est sûre : en refermant ce livre, on ne peut s'empêcher de poursuivre une profonde méditation intérieure ${ }^{19}$.

Il est intéressant de remarquer d'abord la référence au Dernier des Justes, qui rappelle l'intérêt porté notamment dans les milieux catholiques au premier roman d'André Schwarz-Bart, particulièrement dans l'Hexagone. L'auteur semble discrètement suivi par la communauté chrétienne depuis $1959^{20}$ : en Guadeloupe, G. Lemerdy livrait ainsi dans le numéro du 3 mars 1967 de la revue Clartés, elle aussi explicitement tournée vers l'actualité religieuse, une longue critique du Plat de porc aux bananes vertes. En 1972, la courte critique littéraire de R. Wendling dans Églises de Guadeloupe vient confirmer cette intuition ${ }^{21}$ : le premier roman d'André Schwarz-Bart est en effet présent en filigrane tout au long du passage. La mention d'une « croisade contre le racisme » dans Le Dernier des Justes révèle la volonté assumée de créer un lien entre ces deux romans. De même, lorsqu'il est précisé que l'auteur "ne porte pas de jugement », sourd à ce qui peut être compris comme une comparaison qui vise à rassurer le lecteur : La Mulâtresse Solitude de 1972 se distinguerait en cela du roman qui avait tant fait parler de lui en 1959. R. Wendling fait preuve d'une lecture enthousiaste et sensible en soulignant les paradoxes qui irriguent La Mulâtresse Solitude et tout particulièrement la dualité du personnage principal: Solitude, être à la fois fantomatique et charismatique. Contrairement à la notice de Guadeloupe 2000, celle de Églises de Guadeloupe s'attarde sur les souffrances des êtres réduits en esclavage plus que sur leurs origines ou la révolte et le combat des marrons en Guadeloupe. Un discret hommage est rendu à André Schwarz-Bart et à ses talents d'écrivain: sont appréciés le ton et les images; la construction des personnages fait aussi l'objet d'une attention particulièrement laudative de la part du critique.

12 Le roman d'André Schwarz-Bart se donne ainsi à voir, dans des proportions très modestes, dans la production journalistique de 1972, et ce grâce à l'attention que porte la communauté chrétienne à son auteur depuis 1959. Il est ainsi l'un des rares romans dont la parution est médiatisée, dans une période au cours de laquelle la plus grande partie de la population lettrée guadeloupéenne semble ne se préoccuper de littérature contemporaine que si elle fait écho aux revendications politiques de l'époque ${ }^{22}$. Il importe de noter qu'aucun des articles précédemment cités ne contient une quelconque attaque personnelle à l'encontre d'André Schwarz-Bart, de sa couleur de peau, ni d'interrogations sur son appartenance à la communauté antillaise, ni sur sa légitimité à écrire au sujet de l'esclavage : la fiction occupe la plus grande partie des notices, et, lorsqu'il est mentionné, le travail littéraire de l'écrivain est apprécié. Certes, l'ancrage chrétien des journalistes cités explique leur intérêt pour André SchwarzBart ; il est sans doute significatif qu'ils s'attachent à relayer, et ce de manière positive, 
la parution de ce roman. Quoique leur orientation parfois peu critique de la période coloniale et de ses apports, de même que le faible tirage de leurs publications puissent remettre en question leur capacité à représenter l'opinion de l'ensemble de la communauté antillaise, les réactions qu'elles expriment ne questionnent pas la légitimité de l'écrivain.

Le petit nombre de publications relevé ainsi que la ligne éditoriale très spécifique des deux revues pourraient nourrir l'idée qu'il aurait existé une autre réception: celle d'une presse qui n'aurait pas été conservée aux archives départementales, ou celle, informelle, d'un territoire à très forte tradition orale comme la Guadeloupe. Cependant, si avait été ouvertement exprimée en Guadeloupe, à la publication du roman, en février 1972, l'hostilité de plus en plus communément admise aujourd'hui ${ }^{23}$, ces deux articles publiés trois à sept mois après la publication du roman ne manqueraient pas, selon nous, d'en faire état: on noterait un style d'écriture sur la défensive, cherchant à contrer des idées reçues ou des arguments qui auraient été avancés. Or, on ne décèle, à la lecture desdits articles, aucune trace d'une réception qui les aurait précédés: ils présentent un nouveau roman dont peu de lettrés ont entendu parler.

14 Ainsi, aucun élément ne vient, au cours de l'année 1972, étayer la thèse d'un " procès en légitimité » intenté à André Schwarz-Bart à la parution de La Mulâtresse Solitude ${ }^{24}$. En adéquation avec ce qu'évoque Simone Schwarz-Bart dans son discours de réception du prix Carbet à Goyave ${ }^{25}$, étant donné la nature élogieuse des quelques critiques alors formulées, il est possible qu'André Schwarz-Bart ait été, non seulement apprécié de ses contemporains, mais aussi entièrement intégré dans la Guadeloupe qu'il avait choisie comme terre d'accueil. On pourrait alors penser que davantage d'articles y auraient été consacrés, comme c'est le cas généralement quelques mois après la parution d'un roman ; toutefois, la sortie du roman de Simone Schwarz-Bart, en octobre 1972, semble éclipser dans la presse guadeloupéenne celui d'André Schwarz-Bart: les pluies et les vents qui s'abattent sur la jeune écrivaine occultent, un temps, la célèbre Solitude.

\section{Pluie et Vent sur Télumée Miracle (1972) : défense médiatique d'un roman apprécié}

15 Le $1^{\text {er }}$ octobre 1972, huit mois après la parution de La Mulâtresse Solitude, les éditions du Seuil présentent au public le roman de Simone Schwarz-Bart, Pluie et Vent sur Télumée Miracle. Comme pour La Mulâtresse Solitude, on observe très peu de mentions du roman dans la presse guadeloupéenne lors de sa sortie.

Simone Schwarz-Bart, dans son discours de réception du prix des Lectrices de Elle en mai 1973, souligne que le roman touche les Guadeloupéens, flattés de voir dépeints le monde paysan, ses pratiques et ses coutumes:

On m'a dit que le livre était très bien accueilli dans le milieu intellectuel c'est-à-dire que les professeurs l'aimaient, les docteurs, les étudiants; et puis au cours de cette signature, beaucoup de paysans sont venus vers moi, m'ont fait leur écrire sur la paume de leur main le prix de mon livre; ont été dehors, se sont motivés, sont revenus vers moi, m'ont demandé : est-ce que tu es sûre qu'il n'y a pas de mots difficiles jusqu'au bout? que nous pourrons le lire? Parce que ces gens ne disposent que d'un vocabulaire très réduit leur permettant tout juste de lire; et cela m'a énormément touchée ${ }^{26}$.

17 Ce discours montre de plus que, si la presse guadeloupéenne n'évoque pas ce roman immédiatement à sa sortie, il semble que, dans certains milieux, on en parle dès la fin 
de l'année 1972 et le début de l'année $1973^{27}$. Simone Schwarz-Bart évoque une " signature ", celle, sans aucun doute, du 5 janvier 1973 lors d'une rencontre organisée à l'UER ${ }^{28}$ Lettres du Centre universitaire Antilles-Guyane, dans les locaux de l'ancien lycée Carnot à Pointe-à-Pitre. Sur l'affiche présentant cette rencontre, il est en effet précisé : « à l'issue de la causerie, l'auteur signera ses œuvres ».

\section{FIg.1 : AFfiCHE DE PRÉSENTATION DE LA RENCONTRE DE CARNOT}

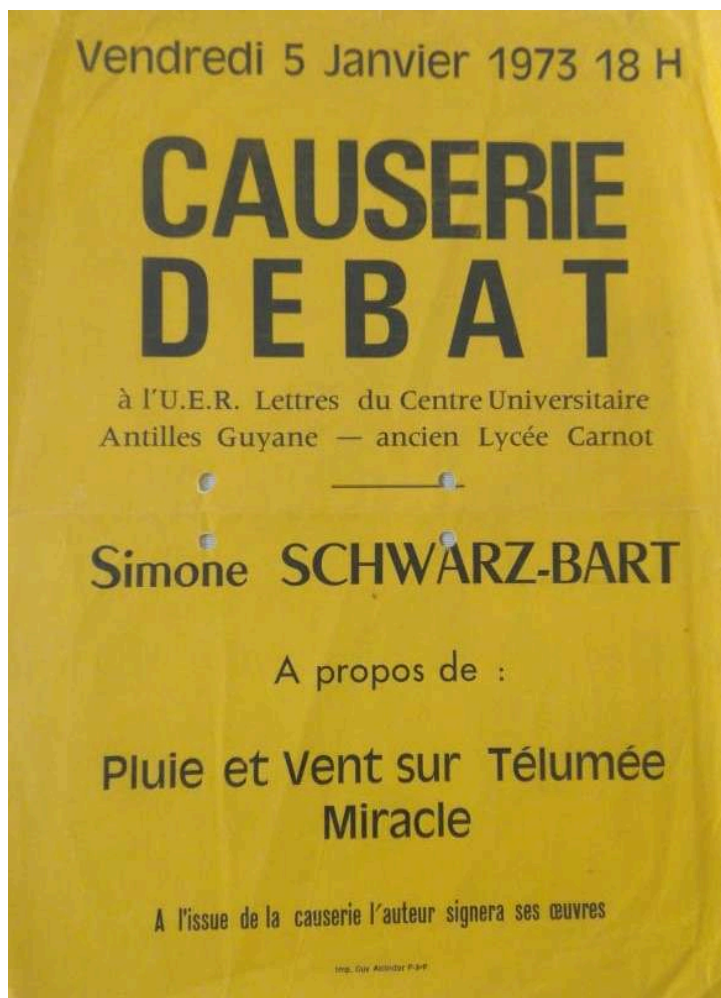

Reproduction avec l'aimable autorisation de Mme Simone Schwarz-Bart

En février 1973, le roman comme cette rencontre sont, pour la première fois, évoqués dans La Vérité, journal qui se présente comme un «organe de combat du peuple guadeloupéen diffusant des informations politiques et sociales» et comme un « journal anticolonialiste ». Ce journal ne contient aucune rubrique littéraire: pourtant, on trouve dans ce numéro une critique précise du roman Pluie et Vent sur Télumée Miracle qui vient partiellement confirmer les propos de Simone Schwarz-Bart quant à l'engouement populaire que suscite son roman :

[...] L'auteur reproduit des témoignages qui dénotent un souci profond de photographier la réalité Guadeloupéenne (sic) dans sa vivante authenticité. Des touches discrètes mais combien pittoresques évoquent le passé esclavagiste de notre peuple. Télumée plie sous le poids de la misère et des vicissitudes de l'existence mais jamais elle ne rompt. Femme courageuse, femme forte, héritière d'une longue tradition de lutte et de résistance à l'adversité que symbolise la puissante, la merveilleuse Reine Sans Nom, Télumée refuse de verser dans le désespoir. Sûre de son bon droit et soucieuse de sauvegarder sa dignité, elle conjure le démon sous quelque forme qu'il se présente et «Monsieur Desaragne se rejette en arrière l'air hagard ». Au terme de sa vie, Elie revient " quémander une parole de réconfort ». Télumée reste fidèle à elle-même, le combat qu'elle livre prend parfois des proportions épiques, toujours la tragédie la guette. Battue, anéantie, trahie, traînée dans la fange, abandonnée, elle reste elle-même, elle a souffert parce qu'elle 
est noire, elle a souffert parce qu'elle est femme, elle a souffert parce qu'elle appartient à cette classe miséreuse inaltérablement soumise au joug de l'exploiteur, mais avec obstination, avec héroïsme elle résiste. "Je mourrai là comme je suis, debout dans mon jardin, quelle joie ", dit-elle.

Le ton est remarquable de simplicité. L'œuvre regorge d'une telle poésie dramatique et lyrique. Le roman se déroule au rythme des événements présentés et des réflexions qu'ils suscitent chez les personnages ${ }^{29}$. [...]

Vie aliénante, dévirilisante de ces hommes ivrognes et violents, vie abêtissante et dissolue de ces négresses qui toujours se dressent. À mesure que nous suivons les péripéties de la vie de Télumée, il nous prend l'envie d'entonner cette merveilleuse chanson créole ${ }^{30}$.

19 Cet article est intéressant à trois niveaux. Il offre d'abord une critique relativement poussée du roman de Simone Schwarz-Bart, dont la portée sociale est remarquablement mise en avant et constitue la plus grande partie de la note de lecture. En accord avec la ligne éditoriale de La Vérité, Pluie et Vent sur Télumée Miracle est présenté comme un roman engagé, peinture sociale des rapports de domination instaurés par la colonisation et encore d'actualité, non seulement à l'époque où évolue le personnage de Télumée, mais peut-être aussi en 1973 lorsque le roman paraît. En effet, pour le ou la journaliste, la fiction ne semble pas ancrée dans une époque révolue : il félicite l'écrivaine d'avoir réussi à capter un réel contemporain, ce qui lui permet de souligner sa propre ligne éditoriale. De plus, il relève des « touches discrètes mais combien pittoresques [qui] évoquent le passé esclavagiste [du] peuple», références à l'histoire douloureuse et traumatique des Guadeloupéens.

L'éloge littéraire du roman ne fait, quant à lui, aucun doute : la sensibilité du critique est touchée et la fougue avec laquelle il s'attache à encenser le roman est marquée par des paradoxes révélateurs, comme la «simplicité » d'une «œuvre [qui] regorge d'une telle poésie dramatique et lyrique ». Cette impression est renforcée par l'évocation de la tragédie et de l'épique qui semblent ici fonctionner non plus comme des marqueurs de genres littéraires, ou des indicateurs tonaux, mais bien plus comme des modalisateurs visant à accentuer le propos laudatif du journaliste.

21 Cette lecture, la première qui évoque le roman dans la presse, est donc favorable au roman, sur le plan sociopolitique et littéraire. En creux transparaissent les critiques contre lesquelles s'élève le journaliste, et qui constituent le troisième axe d'analyse de cet article. En effet, dès les premières lignes, la critique s'insurge contre les opinions émises lors de la rencontre au lycée Carnot:

Pourquoi ne pas le dire, nous avons ouvert le livre de Simone Schwarz-Bart avec quelque méfiance. Mais nous avons été rassurés dès les premières pages. [...] Les tournures sont familières et c'est fort injustement que la forme de Simone SchwarzBart a été attaquée au cours de la conférence débat organisée par le Conseil de l'Unité de l'U.E.R. - Lettres. La mauvaise querelle qu'on a faite à la romancière ne laisse pas de nous surprendre $[. . .]^{31}$.

Notre analyse exhaustive s'arrêtant en février 1973, elle nous permet d'affirmer que cet article fut le premier article publié au sujet du roman de Simone Schwarz-Bart. Nous ne pouvons cependant affirmer que ce soit le seul de l'année 1973, même si nos explorations tendent en faveur d'une réception timide sur le modèle de La Mulâtresse Solitude, et révèle, quoi qu'il en soit, l'absence d'une forte vague de réception de l'ouvrage. La presse, qui se réclame ici comme du côté du "peuple guadeloupéen » et donc en adéquation avec l'opinion populaire, s'opposerait ici à des réactions négatives émanant d'instances universitaires, préfigurant un phénomène très marqué dans les 
années 1980 en Guadeloupe : la scission entre le monde "populaire» et le monde « universitaire » que souligne notamment l'historien J. Dumont ${ }^{32}$.

\section{3, la douloureuse réception universitaire antillaise}

En janvier 1973, a lieu ce que nous nommons « la rencontre de Carnot»: celle-ci nous invite immanquablement à explorer dans cet article, au moins partiellement, l'aspect universitaire de la réception des Schwarz-Bart. Cet aspect n'a pas encore fait l'objet d'une étude approfondie : il nous semble cependant possible, au vu des documents que nous avons pu consulter, d'amorcer ici quelques réflexions. Nous pourrions ainsi faire l'hypothèse qu'une petite faction d'universitaires guadeloupéens auraient contesté la légitimité de La Mulâtresse Solitude comme ils l'ont fait pour Pluie et Vent sur Télumée Miracle.

Dès le début des années 1970, dans le monde universitaire guadeloupéen, les réflexions entamées dans le milieu militant depuis le milieu des années 1960 sont conceptualisées et relayées par les intellectuels: qui sommes-nous lorsque nous nous disons " guadeloupéens »? Comment définir précisément cette identité en construction? Sans exploiter encore les réflexions indépendantistes pourtant déjà bien présentes dans les années 1970, le monde universitaire s'empare de la question identitaire. La " rencontre de Carnot » en janvier 1973, dont fait état La Vérité, marque les débuts d'une prise de conscience littéraire et culturelle qui aura des conséquences décisives sur la manière dont se construiront les jeunes écrivains vivant en Guadeloupe à cette époque.

En 1967-1968, le Centre Études Supérieures de Lettres ouvre et se glisse, dans l'enseignement supérieur, à la place jusque-là occupée par l'année de propédeutique proposée à l'École normale de Pointe-à-Pitre. Il offre alors une formation plus large et plus complète à ceux qui souhaitent se former aux études littéraires. En 1971, il déménage et accueille les étudiants dans le bâtiment du lycée Carnot à Pointe-à-Pitre. À la même époque, l'École normale s'installe sur le Morne Ferret aux Abymes. Dans ces deux institutions s'épanouissent les intellectuels ${ }^{33}$ qui deviendront parmi les plus influents de la Guadeloupe. Ceux-ci contribuent à faire connaître, dès sa parution, le dernier roman de Simone Schwarz-Bart aux étudiants et au grand public lettré : souhaitant asseoir leur présence dans les débats de l'époque, et profitant d'un court séjour de Simone et d'André Schwarz-Bart en Guadeloupe ${ }^{34}$, ils organisent une rencontre avec « la compatriote qui a manqué de peu le prix Goncourt ${ }^{35}$ » autour de son actualité littéraire la plus proche : la parution de Pluie et Vent sur Télumée Miracle. Notre exploration se fonde essentiellement sur les témoignages oraux que nous avons pu réunir puisque la rencontre n'a été ni retranscrite, ni rapportée dans les médias de l'époque. On en trouve une brève mention dans l'ouvrage d'Edmond Dupland ${ }^{36}$; mais $L a$ Vérité nous en livre indirectement la retranscription la plus complète :

[...] Les tournures sont familières et c'est fort injustement que la forme de Simone Schwarz-Bart a été attaquée au cours de la conférence débat organisée par le Conseil de l'Unité de l'U.E.R. - Lettres. La mauvaise querelle qu'on a faite à la romancière ne laisse pas de nous surprendre car est-ce bien utile d'avoir recours à des créolismes quand Télumée sait si bien répondre à Elie que « Le couteau seul sait ce qu'il y a dans le cœur du giraumon»? Quel créolisme serait à la fois plus pittoresque et plus familier que cette réponse de Télumée à Amboise : "Rien même Amboise, c'est s'enfuir que je la vois s'enfuir »?

Quant aux divagations prophétiques de ces extra-lucides qui ont prétentieusement déterminé les limites de la création littéraire de notre compatriote, nous préférons 
les ignorer car il est ridicule et insensé d'interpeller un écrivain en ces termes: « voilà ce que vous auriez dû faire pour améliorer votre roman. » Bref, évitons de polémiquer, nous aurions beaucoup trop à dire sur la qualité et le contenu de certaines interventions. Nous disons que seule une Guadeloupéenne authentique pourrait drainer si totalement les frémissements de la réalité Guadeloupéenne (sic). Certes, Simone Schwarz-Bart n'a pas atteint la classe de Jacques Roumain qui lui, a su allier les ressources de la langue savante et celle de la langue populaire, mais la simplicité du style de notre compatriote permet d'appréhender toute la vérité et la permanence de la misérable vie que mènent les pauvres nègres de la Guadeloupe ${ }^{37}$. interpellations dont Simone Schwarz-Bart a fait l'objet de la part du public, essentiellement composé d'étudiants et de professeurs, et de quelques mondains, qui seraient venus assister à la conférence. Simone Schwarz-Bart souligne, quant à elle, la présence de paysans ${ }^{38}$. On ne sait pas exactement combien de personnes ont assisté à cette rencontre ouverte au public: certains des témoins directs que nous avons interrogés ${ }^{39}$ font état d'une salle comble, d'autres se désolent de l'avoir vue vide. Tous, cependant, concordent avec ce qu'en disent l'article de La Vérité et Edmond Dupland, et rapportent une rencontre qui dégénère très rapidement après l'intervention de l'enseignant Fritz Gracchus ${ }^{40}$ :

Également convient-il de déceler dans le mot de «régionalisme » caractérisant incontestablement la poésie guadeloupéenne depuis 1900 environ, une possibilité d'interprétation extensive à l'antillanité [...] Toutefois encore, cette interprétation risque de se heurter à des conceptions individuelles variées, à défaut de définition ou de théorie précise de l'antillanité (ou de l'antillanisme) comme l'atteste cette critique inattendue émise au cours d'un débat sur le roman pourtant typiquement guadeloupéen de Simone Schwarz-Bart, Pluie et Vent sur Télumée Miracle, par un jeune professeur de philosophie : «Votre livre n'a rien d'antillais!» À quoi l'auteur ne pouvait que répliquer : « Dans ce cas je ne suis pas antillaise non plus $! »^{41}$.

L'attaque, qui surprend l'ensemble des témoins ${ }^{42}$, fait porter l'accusation sur deux aspects de Pluie et Vent sur Télumée Miracle: d'abord, son écriture que l'on déclare, au moins jusqu'en $1979^{43}$, asservie aux codes linguistiques, stylistiques et culturels de l'ancien colonisateur français. Ensuite, la paternité du roman de Simone Schwarz-Bart : si le roman n'est pas «antillais ", seul quelqu'un de déconnecté de la réalité du monde antillais ou quelqu'un qui n'aurait pas suffisamment vécu aux Antilles pourrait l'avoir écrit. Deux postures dominent au cours de la rencontre : la première accuse le roman d'exotisme et Simone Schwarz-Bart d'être une " petite-bourgeoise ${ }^{44}$ » qui ne vit plus en Guadeloupe depuis des années; la seconde désigne André Schwarz-Bart comme le véritable auteur du roman, avançant pour preuve certaines marques stylistiques similaires à celles qui apparaissent dans Le Dernier des Justes ${ }^{45}$. Quoique nous n'en ayons pas retrouvé de trace écrite, il semble, aux dires des témoins directs que nous avons interrogés, que seuls quelques universitaires, dont Fritz Gracchus, ont alors lu Pluie et Vent sur Télumée Miracle: sans que nul dans le public puisse leur répondre, ils se seraient donc employés à commenter un style qui ne serait pas " original », à souligner une série de clichés sur le monde paysan guadeloupéen mais aussi à pointer du doigt les défauts dans la narration... ou les prouesses stylistiques dignes d'un Prix Goncourt, qui, elles, viendraient prouver une autre paternité du roman. La légitimité des deux auteurs est donc remise en cause par quelques-uns, dont les attaques sont renforcées par un machisme latent à l'égard de Simone Schwarz-Bart ${ }^{46}$. 
Comment André et Simone Schwarz-Bart ont-ils perçu le débat houleux de Carnot ? Nos témoins décrivent un couple mortifié, une écrivaine paralysée dont la timidité apparaît aux yeux de ses détracteurs comme une nouvelle preuve de son impossible auctorialité. Aucune note n'a encore été retrouvée à ce sujet parmi les manuscrits d'André SchwarzBart; Simone Schwarz-Bart, quoique sa parole se libère peu à peu, se montre encore réticente à raviver les tensions. Il est cependant intéressant de remarquer qu'en 2015 elle témoigne au sujet de cette conférence sous la plume de Nathalie Levisalles :

Ils lui expliquent, se souvient-elle, que «c'est mauvais pour le combat indépendantiste que Pluie et Vent ait du succès en France. C'est suspect que les Blancs te valorisent. Si tu étais quelqu'un de bien, tu aurais écrit en créole. - C'est écrit en créole, même si vous ne le voyez pas. - Tu es une petite-bourgeoise, comment tu te permets de parler du peuple ? » André s'est levé : « J'espère qu'il n'y a dans cette assemblée aucun jeune Antillais qui souhaite écrire, parce qu'il serait dégoûté à jamais. » Fin de leurs relations avec les indépendantistes et début de la traversée du désert ${ }^{47}$.

Si la publication par les Éditions du Seuil est effectivement déjà la marque d'un intérêt parisien pour le roman, il est surtout probablement ici question du prix Goncourt de 1972, qui compte Pluie et Vent sur Télumée Miracle parmi ses potentiels lauréats, dont le déroulé est suivi avec attention, semble-t-il, dans le milieu antillais ${ }^{48}$. Une voix manque cependant : le roman n'est pas primé, et nombreux sont ceux qui s'insurgent contre cette décision. Le couronnement du roman de Simone Schwarz-Bart en mai 1973 par le prix des lectrices de Elle, et les diverses émissions télévisées et radiophoniques qui suivront, documentant la vie et l'œuvre du couple Schwarz-Bart, confirmeront cependant quelques mois plus tard qu'en "France ", Pluie et Vent sur Télumée Miracle a $\mathrm{du}$ « succès ». Simone Schwarz-Bart, dans cet entretien, évoque aussi la question du créole; tous nos témoins ont formellement démenti qu'elle se soit posée de manière aussi claire. La revendication d'une littérature en créole (langue qui n'est pas encore normalisée ni valorisée dans l'enseignement universitaire) ne semble pas présente dans les rencontres littéraires avant $1975^{49}$ : il n'entre pas au nombre des critères qui permettent de juger de la pertinence d'une œuvre littéraire au début des années 1970. En effet, est-il nécessaire de rappeler que, même au sein du monde universitaire antillais, en Guadeloupe notamment, c'est essentiellement la prose d'Aimé Césaire, le normalien, qui est alors étudiée lorsqu'on daigne s'intéresser aux romans de la littérature antillaise ? En 1973, aurait été déplorée l'absence de « créolismes » dans Pluie et Vent sur Télumée Miracle, de teintes créoles, qui ne sont pourtant pas encore unanimement considérées comme des marques de valeur par les universitaires; mais il n'est pas question de blâmer les écrivains qui n'écrivent pas en créole avant le début des années 1980. On peut donc penser, sur ce dernier point, que les attaques auxquelles fait référence Simone Schwarz-Bart auraient plutôt été formulées lors d'une autre rencontre qui aurait eu lieu à Capesterre-Belle-Eau en 1980, où il lui aurait été clairement reproché de n'avoir pas écrit en créole ${ }^{50}$. La phrase qu'aurait publiquement prononcée André Schwarz-Bart nous a, quant à elle, été confirmée par tous les témoins que nous avons pu interroger : «à la fin, il prend la parole pour déplorer le climat de cette conférence, et reproche au milieu intellectuel de ne pas encourager les jeunes auteurs ${ }^{51}$ »; "André, lui, était amer: il dit que s'il y a des jeunes écrivains dans le public, cette soirée a détruit chez eux tous les germes de création $!^{52}$ ». Cette dernière remarque est significative et peut être interprétée soit comme l'expression d'une réflexion à l'œuvre dans sa décision d'entrer en silence, soit comme une vision prophétique de ce qui va vraiment avoir lieu dans les années suivantes, lorsque sa 
production littéraire peinera à voir le jour. Le dégoût qu'il exprime peut aussi être un acte de compassion pour Simone, qu'il a si activement encouragée à écrire ${ }^{53}$.

Les écrivains sont visiblement désemparés face à ce public constitué de proches, de soutiens venus applaudir leur plume, mais aussi d'intellectuels qui veulent, peut-être maladroitement, générer un débat et une discussion littéraire. Il ne nous semble pas, cependant, que les attaques de Carnot aient véritablement pu générer un silence littéraire : d'abord parce qu'elles s'adressent avant tout à Simone Schwarz-Bart, qui publiera Ti-Jean l'Horizon moins de sept ans plus tard; nous n'avons pas retrouvé, à ce stade de nos recherches, de critique universitaire négative émise depuis les Antilles en 1972, attaquant la légitimité d'André Schwarz-Bart. Certes, cette critique pourrait aussi être comprise, comme le suggère Gyssels, dans un " silence », une indifférence à l'égard des auteurs, qui n'est pas « réparée » par l'attribution du prix Carbet en $2008^{54}$. Il nous semble cependant qu'elle aurait aussi été explicitement formulée. Ensuite parce que la réception universitaire, aussi douloureuse qu'elle soit, ne reflète pas, nous l'avons vu, ce qu'aurait été la réception populaire des romans des Schwarz-Bart ${ }^{55}$. Cette donnée est capitale, car André Schwarz-Bart comme Simone Schwarz-Bart tiennent à distinguer les deux types de lecteurs à qui ils proposent leurs romans, à qui ils "serrent la main ${ }^{56}$ " par le biais de l'écriture, et semblent s'attacher à se focaliser sur l'opinion de leur lectorat non universitaire: nous en voulons pour preuve le fait que tous deux continuent d'écrire et de publier même après une réception critique intellectuelle manifestement négative de leur premier roman, celle du Dernier des Justes comme celle de Pluie et Vent sur Télumée Miracle.

En 1972-1973, le contexte médiatique dans les Antilles, et tout particulièrement en Guadeloupe, passe sous silence les nouveautés littéraires, à moins qu'elles se présentent comme des ouvrages contemporains engagés ${ }^{57}$. La presse se consacre en effet essentiellement à des questions sociales et s'emploie à dénoncer les inégalités qui subsistent et se creusent depuis la loi de départementalisation de 1946, entre l'ancienne "métropole » et ses « ex-colonies ». La Mulâtresse Solitude ne fait ainsi pas l'objet d'un blessant rejet de la part de la communauté antillaise : si ce n'est dans certains milieux chrétiens qui lui offrent un accueil élogieux, ce roman s'inscrit dans un climat qui n'accorde pas une importance capitale aux actualités littéraires. Il est possible qu'il ait fait l'objet d'attaques isolées, émises dans l'intimité d'un cercle restreint de connaissances d'André et de Simone Schwarz-Bart; mais nullement d'un "procès " mené à l'échelle de l'archipel. Si procès en légitimité il y a, il semble qu'il soit plutôt dirigé contre Simone Schwarz-Bart que contre André Schwarz-Bart: nos recherches actuelles, certes larges mais non exhaustives, n'ont révélé à ce jour, aucune réaction universitaire, en Guadeloupe, à la parution de La Mulâtresse Solitude. Comment, alors, comprendre le silence de son auteur? 
Fig. 2 : Le lycée Carnot à Pointe-à-Pitre

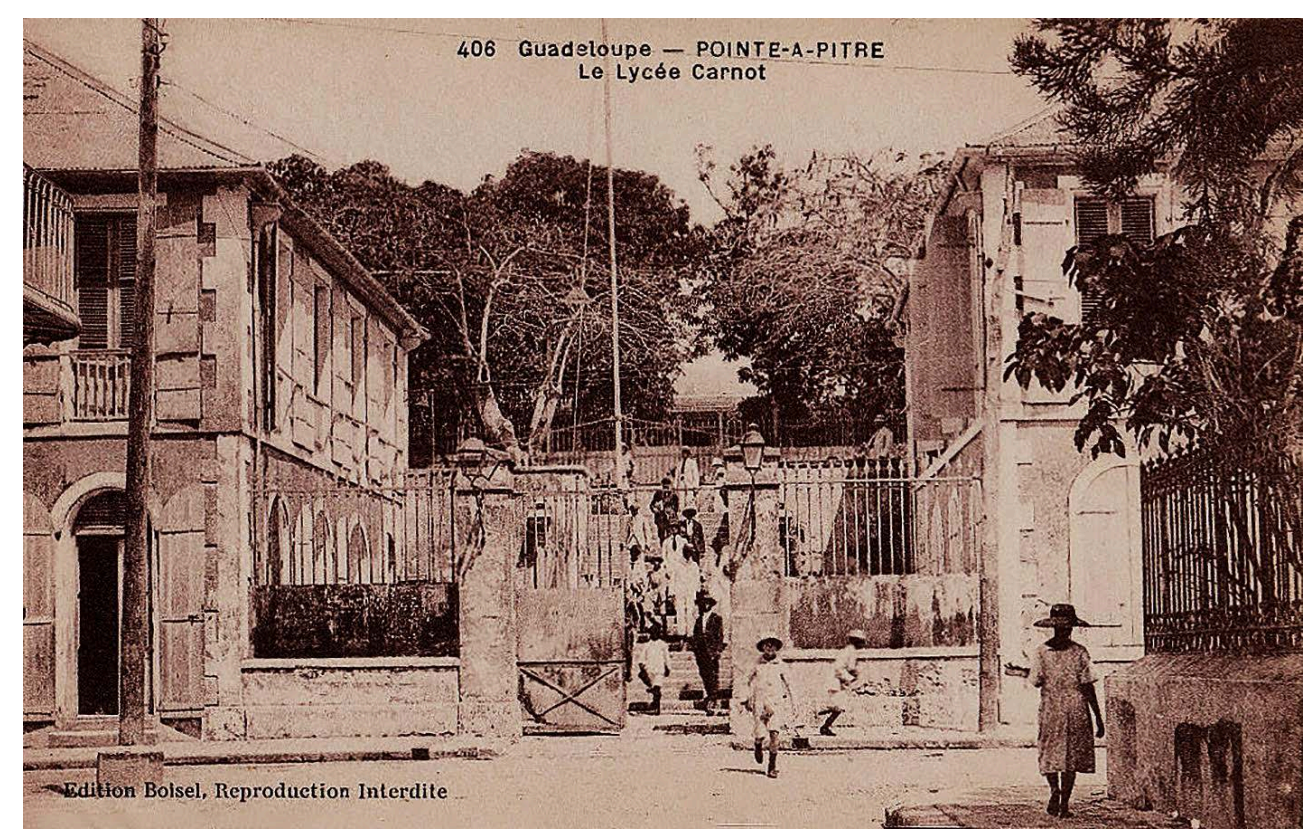

Charles Boisel

\section{Comment expliquer le silence d'André et de Simone Schwarz-Bart?}

En 2015, dans l'avant-propos de L'Ancêtre en Solitude, Simone Schwarz-Bart retrace les étapes du silence d'André Schwarz-Bart :

Le livre [La Mulâtresse Solitude] paraît en 1972, et c'est une rupture dans le déroulement prévu du cycle. Il paraît à la grande époque de l'« intimité ethnique ». C'est le lynchage: le procès en légitimité. [...] André Schwarz-Bart pense que ce livre n'est nécessaire à personne. Et il entre en silence sans explication, sans hésitation, sans explosion ${ }^{58}$.

Ce court témoignage génère peut-être la réflexion de J. Garcin ${ }^{59}$ : il semble en effet que ce soit la première occurrence de l'expression "procès en légitimité ». Il en ressort, selon nous, un bref témoignage sur ce qui a dû marquer, personnellement, le couple ; à savoir la pression qui pèse, à l'époque, sur les mariages dits " mixtes ", entre personnes blanches et personnes de couleur : cette pression prédomine tout particulièrement dans les milieux religieux, et tend à s'étendre, au milieu des années 1970 et surtout au début des années 1980, à l'ensemble de la société. Si elle touche personnellement le couple d'écrivains, il ne nous semble pas, cependant, qu'elle ait pu motiver un réel "procès en légitimité » à l'échelle de la Guadeloupe tel que l'on a tendance à le dépeindre aujourd'hui. Une lecture attentive des mots de Simone Schwarz-Bart révèle deux indices d'importance capitale qui viennent appuyer cette hypothèse. Le premier, sur lequel nous reviendrons, est l'importance pour André Schwarz-Bart que son roman ait une utilité sociale ; le second est l'absence de déclaration, publique ou personnelle, de la part de l'écrivain pouvant justifier son entrée en silence. Nul, à part lui-même, ne sait pourquoi il cesse de publier. 
Examinant la réception médiatique de 1972, rien ne nous semble prouver qu'André Schwarz-Bart ait fait l'objet d'un «lynchage ». Celui-ci ayant peut-être eu lieu, comme pour Pluie et Vent sur Télumée Miracle, dans les milieux universitaires, il importe d'examiner plus précisément le contexte universitaire de 1972: est-il propice à des attaques qui délégitimeraient la qualité littéraire de La Mulâtresse Solitude par la couleur de peau de son auteur? Sinon, peut-être devrions-nous alors avancer l'hypothèse d'une seconde vague de réception, populaire ou universitaire, plus tardive, qui viendrait attester un "procès en légitimité ", ou émettre d'autres pistes d'explication au silence littéraire d'André Schwarz-Bart.

\section{Penser la littérature antillaise en 1972 : les balbutiements d'un champ d'étude en construction}

Au sein des cercles qui s'attachent à l'étude universitaire de la littérature antillaise, André et Simone Schwarz-Bart font, dès 1972, l'objet d'études. La plus célèbre d'entre elles, à laquelle fait notamment référence $\mathrm{K}$. Gyssels ${ }^{60}$, est la lecture que propose Maryse Condé de Pluie et Vent sur Télumée Miracle, et, dans une moindre mesure, de La Mulâtresse Solitude, dans la revue Présence Africaine ${ }^{61}$. Cette lecture est ponctuée par un bref clin d'œil à Un plat de porc aux bananes vertes, connivence avec le lecteur qui nous permet d'avancer que ce premier roman co-signé était, lui aussi, connu du monde universitaire auquel appartenait Maryse Condé. Le compte-rendu de lecture de la critique de Présence Africaine qui deviendra à son tour une écrivaine renommée suscite quelques réflexions. Il nous semble en effet plus ambigu qu'il n'y paraît: sous une critique acerbe et ironique, Maryse Condé survole le sujet des romans et l'identité de ses auteurs, pour mieux s'attacher aux questions lancinantes de la définition de l'écrivain antillais, et de la place qu'occupe ce qu'elle nomme l'« exotisme » dans les romans antillais ${ }^{62}$. Elle juge ainsi que :

[...] pour nous La Mulâtresse Solitude d'André Schwarz-Bart était un élégant produit exotique très visiblement destiné au marché non local et qui, à ce titre, n'avait pas nécessité notre critique ${ }^{63}$.

Et

Que manque-t-il à Pluie et Vent sur Télumée Miracle? Peut-être simplement ce que nos frères afro-américains appellent : « Soul $\aleph^{64}$.

Maryse Condé s'attarde sur les manques dont semblent souffrir les romans: superficiels, sont-ils vraiment aptes à refléter la réalité antillaise, ou se contentent-ils de jouer sur les clichés attendus par un public de lecteurs qui ne connaissent de la Guadeloupe que les images trompeuses de paysages tropicaux? Ce qui est néanmoins certain, c'est qu'aux yeux de la critique de Présence Africaine qui deviendra son tour une écrivaine renommée, la couleur de peau de l'auteur ou son appartenance communautaire n'entrent pas en ligne de compte de la définition de l'exotisme: en effet, à peine quelques mois plus tôt, nombreux sont les écrivains natifs de Guadeloupe à qui Maryse Condé reproche, entre autres, de cultiver les clichés exotisants. Son article sur l'ouvrage de Jack Corzani ne laisse aucun doute sur ce point :

Que sont les Antilles?

Pour y répondre, il ne faut pas garder les yeux fixés sur une poignée de privilégiés ou de semi-privilégiés, même et surtout, quand ils clament leur appartenance «à la Race de Nuit » (Florette Morand) car ils font de leur Négritude un jeu exotique et indigne, qu'ils dénoncent aussitôt par la totale acceptation de la culture française ${ }^{65}$. 

marqués à la fin des années 1960 et intime aux " acteurs de se "positionner" dans des champs dont l'autonomie est de plus en plus relative ${ }^{68} "$, n'est de prime abord pas suivi par les universitaires guadeloupéens, ceux-ci s'interrogent néanmoins sur ce que serait et devrait être la littérature antillaise que l'on commence tout juste à étudier ${ }^{69}$. Les éditoriaux de la revue CARE prouvent dès 1974 cette ambivalence, entre refus de se laisser dicter des définitions par des mouvements autonomistes qui interrogent l'identité antillaise, désir de dévoiler la qualité littéraire de productions trop longtemps négligées, et tendance à les évaluer au regard de critères politiquement fantasmés et dissociés du réel. En Guadeloupe, au début des années 1970, se développe donc une tendance particulièrement ancrée dans les milieux lettrés une dizaine d'années plus tard : les études littéraires fustigent et méprisent les «doudouistes» qui jouent sur l'exotisme, refusant d'aborder les véritables problématiques sociales et politiques contemporaines. La plus grande partie des intellectuels antillais encense la prose d'Aimé Césaire, de Cahier d'un retour au pays natal ou de Ferrements, vindicative, enflammée, ou encore celle de Vincent Placoly, auquel la revue $C A R E^{70}$ consacrera un numéro spécial. La prose de Simone Schwarz-Bart est ainsi accusée de jouer le jeu des «doudouistes », du folklore, comme le soulignent d'abord Maryse Condé ${ }^{71}$, puis, en 1975, Caroline Oudin-Bastide :

Cette vision du monde qui se dégage de Pluie et Vent n'est pas née dans l'imagination de S. Schwarz-Bart. Elle correspond à une conception idéologique certainement vivante dans la paysannerie [...] Mais, consciemment ou inconsciemment là n'est pas le problème, $\mathrm{S}$. Schwarz-Bart rompt avec la réalité en renforçant par tous les moyens cette idéologie. [...]

En se réfugiant dans un monde mythique, elle n'a pas pu ou pas su nous révéler notre monde réel ${ }^{72}$.

Pour les intellectuels de l'époque, souvent citadins, et qui abordent la réalité sociale en y apposant le filtre de leurs lectures marxistes ou communistes, Pluie et Vent sur Télumée Miracle n'est pas un reflet fidèle de la Guadeloupe, et encore moins de ce qui se joue 
dans les campagnes guadeloupéennes. Se refusant à la revendication, à la lutte sociale, le roman serait, en 1975, à la fois une trahison du peuple et une trahison de la littérature, qui se doit d'être engagée. Il est intéressant de voir que, pétris de cette idéologie, ils reprochent à la Simone Schwarz-Bart qui parcourt la campagne de Goyave, magnétophone au bras pour recueillir les mots des paysans qui l'entourent, de ne proposer dans son roman que son fantasme du vécu paysan. Le débat autour de Pluie et Vent sur Télumée Miracle cristallise donc la question de la manière dont un écrivain guadeloupéen doit écrire, et des thèmes qu'il doit, ou non, aborder. Si elles ne sont pas encore aussi élaborées en 1972, on retrouve les premières bases de ce débat dans l'accueil réservé au roman lors de la rencontre de Carnot.

Il faut cependant souligner que la réception de l'époque n'est personnellement hostile ni à Simone ni à André Schwarz-Bart : une certaine critique universitaire touche avant tout Pluie et Vent sur Télumée Miracle. La thèse d'un procès en légitimité intenté à la parution de La Mulâtresse Solitude nous semble donc pouvoir être écartée, et la perspective selon laquelle André Schwarz-Bart aurait immédiatement cessé d'écrire et de publier après la parution de La Mulâtresse Solitude sous le coup d'attaques motivées par un préjugé de couleur serait donc à nuancer. Un silence immédiat de l'auteur après la publication de La Mulâtresse Solitude serait de plus en contradiction avec la réception que l'ouvrage et son auteur reçoivent dans les milieux antillais, parmi lesquels l'écrivain compte de nombreux amis. On en retrouve par exemple des traces dans la volonté d'Yvan Labéjof d'adapter La Mulâtresse Solitude en 1976 au théâtre, avec la participation d'un jeune Patrick Chamoiseau enthousiaste. Il importe aussi de rappeler que Simone Schwarz-Bart déclare en 1979 au micro de Jacques Chancel ${ }^{73}$ qu' « il [André Schwarz-Bart] est en train d'écrire un essai, et un roman juif, qui sont certainement parmi les choses qu'il a écrites de plus belles ", et semble, par-là, préparer la promotion d'un roman en gestation, probablement destiné à la publication. Il nous semble donc que si André Schwarz-Bart prend la décision de ne plus publier, celle-ci est bien postérieure à 1972.

\section{0-1990: une « seconde vague de réception » de La Mulâtresse Solitude?}

42 Si André Schwarz-Bart évoque en 2003 les raisons de son silence ${ }^{74}$, en l'absence d'archives datées de la main de l'auteur, il semble difficile de déterminer précisément le moment où André Schwarz-Bart décide de ne plus publier. Nous en sommes donc réduits à formuler des hypothèses.

L'idée selon laquelle André Schwarz-Bart aurait été accusé de s'approprier à tort l'histoire de l'esclavage malgré sa couleur de peau et son appartenance communautaire nous semble peu fondée, au vu des recherches que nous avons pu mener sur la base de journaux locaux et de leur état aujourd'hui ; nous avons montré que la contestation de sa légitimité est inexistante lors de la parution de La Mulâtresse Solitude en 1972. Des deux auteurs du couple, Simone Schwarz-Bart est celle qui, manifestement, essuie les critiques acerbes et violentes cette année-là. Pourtant, elle ne cesse d'écrire et de publier, et la parution de Ti-Jean l'Horizon en 1979 génère un engouement médiatique. Les critiques négatives guadeloupéennes des années 1970 n'auraient donc pas raison du couple d'écrivains. 
Selon nous, si la couleur de peau ou l'histoire personnelle d'André Schwarz-Bart devient un frein aux yeux de la communauté antillaise, les prétendues attaques qu'il aurait subies seraient peut-être à situer plus tard, dans les années 1980-1990, lors de l'intensification des luttes nationalistes, à l'ouverture de l'« ère de commémoration ${ }^{75}$ " qui marque un tournant dans les perceptions et revendications mémorielles.

Il est ainsi probable que la remise en cause de la légitimité d'André Schwarz-Bart soit même encore plus tardive, et qu'avant le milieu des années 1990, celui-ci ne fasse l'objet d'aucune réelle accusation d'illégitimité basée sur un préjugé de couleur. Cette idée nous semble confirmée à la fin d'une lettre de 1993 : André Schwarz-Bart répond à Susan Dune qui voulait adapter La Mulâtresse Solitude en film et se demandait s'il en était le seul auteur:

PS : Vous me posez une question sur l'auteur : à ma connaissance, je suis le seul et unique auteur de ce texte, y compris la ponctuation et les silences. J'aimerais savoir ce qui motive cette question : à mon avis, il s'agit d'une récente invention, liée à la montée du communautarisme en littérature ${ }^{76}$.

L'étonnement d'André Schwarz-Bart peut être feint. S'il était sincère, il pourrait alors confirmer cette hypothèse d'un renversement radical de perspective au sujet de $L a$ Mulâtresse Solitude qui n'aurait pas eu lieu avant les années 1990 en Guadeloupe. Nous formulons donc l'hypothèse, encore à prouver, d'une seconde vague de réception au début des années 1990, façonnée par un processus de construction mémorielle et identitaire houleux, marqué par la controverse et la polémique. Cette réception ultérieure serait d'une part minée par de nouveaux enjeux politiques et sociaux, et d'autre part grevée par les approches des critiques anglophones des années 1970-1980 comme Thomas R. Edwards ${ }^{77}$ ou le plus controversé Frederick Ivor Case ${ }^{78}$, tous deux cités par Kathleen Gyssels ${ }^{79}$. On retrouve ainsi des notes rédigées par André SchwarzBart après 1985 qui s'interroge sur l'influence de la signature sur la couverture d'un roman :

André répond dans une annotation manuscrite de l'ouvrage ${ }^{80}:$ "Jusqu'où peut mener la manie interprétative ?... La même phrase change de sens, de valeur, d'intérêt littéraire, selon qu'on imagine un auteur blanc ou noir. Misère de la critique littéraire : imposture fondamentale. Comment ces gens lisent-ils ? $»^{81}$.

Une seconde réception, plus tardive donc, peut-être conditionnée par des lectures du reste de la Caraïbe, notamment anglophone, du milieu des années 1980, tendrait à questionner la légitimité et l'auctorialité d'André Schwarz-Bart, en attribuant le roman portant sur l'esclavage à Simone Schwarz-Bart, considérée comme la part "noire ", " antillaise » du couple, là où André Schwarz-Bart serait la part «juive ». La lecture simplificatrice d'un débat complexe aurait suggéré un glissement hâtif : de « un blanc ne peut pas avoir écrit cela » à " un blanc n'a pas le droit d'écrire cela ", il n'y a qu'un pas à franchir, celui de la signification du verbe " pouvoir ».

Des recherches plus poussées sur la réception après 1972 pourront peut-être nous éclairer : une attaque frontale, une série d'articles à charge, auraient pu favoriser la décision radicale de ne plus publier. Cependant, pour l'instant, rien ne semble ici attester que le silence d'André Schwarz-Bart ait pu vraiment être causé par une seconde vague de réception, négative, où il lui aurait été reproché, en tant que «Blanc » ${ }^{82}$ d'avoir écrit sur l'esclavage et de s'être approprié, avec La Mulâtresse Solitude, une histoire qui n'aurait pas été la sienne. Il est possible de justifier cette approche en rappelant qu'il n'hésite pas, en 1967, à exprimer sa colère : 
Dès le début, plusieurs personnes m'ont laissé entendre qu'un homme blanc ne pouvait honnêtement écrire sur les Noirs. Ce genre de réflexion suscitait en moi une révolte que je crois fondée. Il m'a toujours paru - il me paraît encore - que chaque humain peut légitimement parler de tous ses semblables. Les raisons de son silence pourraient alors être plus personnelles: le résultat d'un
choix et d'une réflexion intime, l'aboutissement d'une vie d'interrogations littéraires ${ }^{83}$.

\section{La douloureuse question de la légitimité}

André Schwarz-Bart, s'entretenant avec Francine Kaufmann en 2003 ne nous fournit pas les éléments nécessaires à l'élaboration d'une réponse tranchée sur le sujet :

Récemment, quelques jours avant son soixante-quinzième anniversaire (en mai 2003), il me confiait dans un café de Saint-Germain-des-Prés avoir fait fausse route en essayant de parler d'un autre peuple et de rendre un son juste. Et pourtant, quelle amertume dans cette confidence : il se réjouissait d'avoir ramené à la vie une héroïne guadeloupéenne dont il ne restait que trois lignes dans un récit et à laquelle il avait donné une telle épaisseur que les enfants de Guadeloupe interprètent avec fierté, chaque année, une pièce tirée de son roman. Mais il se désolait que personne n'ait décelé l'architecture complexe de son texte. Il croyait, me disait-il, avoir écrit un livre "réversible", qui, comme un vêtement qu'on retourne, pouvait se lire des deux côtés à la fois : un côté noir et un côté juif. Déjà, dans le Plat de porc, il avait donné des pistes au lecteur, que personne n'avait relevées. Cette incompréhension l'avait conforté dans son refus de publier... mais pas d'écrire ${ }^{84}$.

51 L'amertume d'André Schwarz-Bart ne semblerait donc pas le fait d'une réception virulente de la part du milieu antillais, du moins en Guadeloupe. Sa déclaration à Francine Kaufmann pourrait aller à l'encontre de celle de Simone Schwarz-Bart dans L'Ancêtre en Solitude : André Schwarz-Bart a-t-il vraiment " pens[é] que ce livre n'[était] nécessaire à personne ", alors même qu'il semble conscient en 2003 de l'impact positif de son roman sur la jeunesse guadeloupéenne? Certes, les années ont passé, et la situation a pu évoluer : son lectorat aurait pu être, une vingtaine d'années en arrière, réticent à la démarche entreprise avec $L a$ Mulâtresse Solitude, puis changer de posture au début des années 2000. De même, il déplore une incompréhension générale de la part d'une partie de son lectorat: critiques et peut-être universitaires peinent à cerner la "réversibilité » de son projet romanesque. Rappelons ici que ce sentiment d'incompréhension de la part de l'élite littéraire le hante depuis Le Dernier des Justes.

Il convient de proposer ici deux hypothèses qui nous semblent pouvoir expliquer le silence littéraire d'André Schwarz-Bart. D'abord, celle qui s'appuie sur le mal-être assumé d'un écrivain qui, faisant l'objet d'un premier scandale dans les milieux littéraires parisiens en 1959, autour de la réception du Dernier des Justes, décide que le lecteur, et particulièrement certains milieux intellectuels critiques, est par trop décevant. L'accueil réservé à Pluie et Vent sur Télumée Miracle viendrait alors confirmer les soupçons douloureux d'un écrivain convaincu de se heurter à une série d'incompréhensions de la part de son lectorat : «il se désolait que personne n'ait décelé l'architecture complexe de son texte ${ }^{85} »$. Celui pour qui la littérature est un acte de partage, pour qui « écrire un roman n'a de sens que s'il relie les hommes entre eux ${ }^{86}$ », a peut-être été blessé de se sentir incompris de ceux qui décortiquent mot à mot les romans ou de ceux qui se réservent le droit de juger de la valeur d'un texte littéraire. Son silence serait alors une réaction de franc rejet de la critique littéraire en général, 
trop encline à chercher le scandaleux entre les lignes des romans ou au sein de l'histoire dans laquelle s'inscrit leur naissance.

Il est avéré que les craintes d'André Schwarz-Bart ont toujours porté sur sa légitimité d'auteur : il demande, par exemple, son aval à Aimé Césaire pour Un plat de porc aux bananes vertes, bien avant la parution de ce roman, et s'interroge même sur sa capacité à écrire un roman qui serait «antillais». Il évoque notamment dans un article de 1967 sa difficulté à véhiculer le « parfum des Antilles » dans ses textes :

Je découvris, soudain, que si mon livre respecte la lettre du monde antillais - peutêtre même l'esprit - il en omet le parfum [...]. Rien, me semblait-il, dans mon texte, ne représentait une trahison de mes frères antillais. Rien sauf, précisément, l'absence de cet indicible que je découvrais et qui est la fleur de toute œuvre jaillie d'un terroir, d'un sol spirituel [...]. Je découvris, dans la désolation, qu'il est une seule façon de respecter entièrement l'originalité d'un peuple: c'est d'en faire partie [...]; il m'apparut alors que [...] si j'étais en mesure depuis une dizaine d'années de respirer le parfum des Antilles, de l'apprécier, de le décrire au besoin, il m'était impossible de devenir moi-même parfum ${ }^{87}$.

André Schwarz-Bart se décrit comme en prise avec un « indicible » qu'il peine à cerner et à définir, un " parfum » qu'il cherche, sans y parvenir, à incarner. Le résultat de ces réflexions angoissées sera la co-signature avec sa femme, d'origine guadeloupéenne, Simone Schwarz-Bart, et probablement la décision prise de vivre au cœur de la communauté rurale de Guadeloupe. On saisit alors le découragement qui fut certainement le sien et qui ne cesse de transparaître dans les feuilles manuscrites éparses retrouvées par Simone Schwarz-Bart et Francine Kaufmann après 2006 dans son bureau de Goyave, dont les plus éloquentes sont publiées dans Nous n'avons pas vu passer les jours ${ }^{88}$.

La seconde hypothèse serait donc qu'André Schwarz-Bart, ne se sentant pas légitime, et conscient qu'il était d'une certaine proximité fraternelle avec les Antillais, aurait peutêtre alors cessé de publier par "solidarité ». Le choix du silence serait alors un choix réfléchi et engagé, en accord avec l'engagement politique et humaniste qui marqua sa vie. Convaincu que le texte littéraire n'existe que dans un lien avec le lecteur, qu'il se doit d'avoir un impact dans le monde, et certain qu'il ne parviendra jamais, par sa littérature, à servir les combats politiques et sociaux d'une île en pleine transformation, André Schwarz-Bart aurait pris la décision de laisser la parole à ceux qui se sentiraient plus légitimes que lui pour la porter. Cette hypothèse, prenant en compte le contexte socio-politique de la Guadeloupe de cette époque, nous est inspirée par les remarques de Daniel Maximin ${ }^{89}$, écrivain proche de la famille Schwarz-Bart. André Schwarz-Bart se serait tu pour soutenir la cause des autonomistes et indépendantistes, desquels il était très proche. L'homme se serait donc effacé, dès la fin des années 1980, pour laisser la voix au mouvement de ceux qu'il considère comme ses frères, faisant ainsi preuve de son caractère d'homme, pour qui l'être individuel n'a que peu de consistance, mais aussi d'écrivain, pour qui les écrits doivent avoir un sens et s'inscrire dans un acte de foi et de partage.

56 Ainsi, la publication de l'Hommage à la femme noire ne serait pas l'étonnante expression de la parole fugacement libérée d'un auteur trop longtemps silencieux. Elle s'inscrirait au contraire dans une prise de conscience progressive, de la part d'André Schwarz-Bart, de la nécessité de s'effacer derrière des figures d'écrivain que le public et notamment le public guadeloupéen commence à reconnaître à leur juste valeur. Dès 1979, et plus encore, dès le succès sur la scène guadeloupéenne de Ton Beau Capitaine mis en scène en 
1987 par Syto CAVÉ ${ }^{00}$, Simone Schwarz-Bart semble libérée des jugements qui avaient accablé Pluie et Vent sur Télumée Miracle en 1972: son public et son lectorat se renforcent ; pour André Schwarz-Bart, elle a alors toute légitimité pour porter la voix de la Guadeloupe. Cette légitimité, accrue à la fin des années 1980 grâce à un lectorat universitaire qui lui devient favorable, a toujours semblé une évidence pour l'auteur. Ainsi, après avoir encensé le travail de Simone Schwarz-Bart avec Pluie et Vent sur Télumée Miracle, parlant d'Un plat de porc aux bananes vertes, il déclare :

J'ai rencontré beaucoup de difficultés [...] je ressentais que je restais extérieur [...] or je voulais la vérité, l'authenticité du langage. [...] Connaître le créole, en métropole, ce n'est pas la même chose. Simone connaît le créole comme une langue totale, une expression véritable ${ }^{91}$.

Il nous semble donc qu'André Schwarz-Bart ne cesse pas de publier à la suite d'une ou de plusieurs douloureuses expériences de réception négative qui lui auraient dénié toute légitimité à écrire sur la Guadeloupe. Il n'est en effet pas avéré qu'elle ait pu avoir lieu; et encore moins qu'elle aurait pu avoir un impact sur l'écrivain. Ce qui est certain, c'est qu'il est lui-même, depuis la fin des années 1960, en proie à des doutes qui le minent quant à sa propre légitimité : il ne parvient pas à trouver une exacte adéquation entre ses écrits et sa personnalité. Échaudé par l'incompréhension que rencontre Le Dernier des Justes, et déçu par la compréhension superficielle de ses textes plus tardifs par la critique littéraire, il a conscience du poids qui peut peser sur l'écrivain, et décide, selon nous, de laisser la place à celle qui, pour lui, est l'écrivain légitime des Antilles, Simone Schwarz-Bart, dès l'instant où il sait qu'elle est reconnue comme telle par leurs compatriotes. La littérature qu'elle peut produire lui semble alors apte à avoir un véritable impact positif, notamment pour les luttes sociales et politiques de l'époque. Lui, pour sa part, se réfugierait dans un silence paradoxalement éloquent, refusant l'imposture d'une parole qui ne peut parvenir à vaincre le néant ${ }^{92}$.

\section{Conclusion}

Le silence d'André Schwarz-Bart a-t-il été la conséquence de sa désillusion et de sa déception, ou au contraire celle d'un nouvel engagement politique, personnel et littéraire? Ce dont nous sommes sûrs, c'est que ce silence n'est pas le fruit d'attaques immédiates et unanimes lors de la parution de La Mulâtresse Solitude, ni celui d'un " procès en légitimité » dirigé contre lui par de furieux indépendantistes et qui aurait eu lieu en Guadeloupe dans les années 1970. À l'époque, seule une minorité d'intellectuels, aux discours encore éloignés de ce que seront les injonctions indépendantistes des années 1980, occuperait le devant de la scène et ferait écran en attaquant Pluie et Vent sur Télumée Miracle ${ }^{93}$, et en mettant en exergue les réflexions autour de la nature et de la définition du roman antillais.

Ainsi, nous n'avons pas de preuve, dans la presse, d'une éventuelle mauvaise réception de La Mulâtresse Solitude en Guadeloupe dans les années 1970 et jusqu'au milieu des années 1980. Le peu d'attention que reçoit le roman s'explique en grande partie par le contexte social et culturel dans lequel il s'inscrit. Si André Schwarz-Bart a cessé de publier à cause d'opinions interrogeant publiquement sa légitimité à aborder le thème de l'esclavage, celles-ci n'ont visiblement pas été exprimées avant le milieu des années 1980. Ainsi, André Schwarz-Bart a-t-il cessé d'écrire à cause d'une mauvaise réception antillaise de La Mulâtresse Solitude qui aurait fait écho à celle du Dernier des 
Justes? L'histoire personnelle d'André Schwarz-Bart nous indique qu'il est hanté par une réelle peur d'illégitimité, et par un désir profond de produire une littérature qui puisse apporter un changement. Au vu de ces éléments, il est possible d'émettre l'hypothèse que son entrée en silence serait le résultat d'un questionnement qui lui est propre quant à sa légitimité, renforcé par une compréhension lacunaire, de son point de vue, de ses textes par la critique, et associé à une solidarité, un engagement envers un peuple et une cause qui lui tenait à cœur.

Il nous semble donc que le glissement de la critique actuelle qui s'agace de la réception antillaise des Schwarz-Bart est peut-être un peu hâtivement formulé : soit les SchwarzBart ont effectivement subi, tardivement, des attaques, et ont été mis au ban de la communauté antillaise à cause de la couleur de peau d'André Schwarz-Bart; soit il s'agit d'un détournement abusif des déclarations de Simone Schwarz-Bart qui, la première, donne à voir la "traversée du désert " après la mort de son époux, et notamment lors de la remise du prix Carbet de la Caraïbe en 2008 :

Et puis, ça a été le premier livre après le Dernier des Justes, le premier livre d'André, Un plat de porc aux bananes vertes, qu'on a écrit ensemble; bien entendu, ce n'est pas ce qu'on attendait de lui. Et il le savait. C'était l'image, son image, qu'il cassait. Et, il a été en marge, naturellement, de sa communauté. Puis, ça a été La Mulâtresse Solitude. Et, là, il a... tout simplement été en marge de l'autre communauté qu'il voulait adopter. Il n'est pas bon d'être un précurseur, il n'est pas bon d'être en avance sur son temps. Et, il l'a payé très cher. Et moi aussi. Ça a été la traversée du désert, et cent ans de solitude ${ }^{94}$.

61 On peut remarquer dans ce discours une hésitation au moment d'évoquer la question des marges de la communauté antillaise, que nous avons choisi de retranscrire avec des points de suspension. Simone Schwarz-Bart s'interrompt, peut-être pour trouver les mots justes : "en marge ", est-ce trop fort, ou trop faible, pour décrire ce qui a joué dans le silence littéraire de son époux disparu ? La suite des entretiens qu'elle donnera à partir de 2015 se teinte des mots des journalistes de plus en plus nombreux qui, parfois, s'autorisent à relayer l'idée que les Antillais seraient un peuple raciste et antisémite. La nouvelle vague de réception des Schwarz-Bart dans les années 2010 viendrait peut-être réécrire le récit du silence d'André Schwarz-Bart.

\section{BIBLIOGRAPHIE}

Anonyme, La Vérité, n 76, février 1973.

AITA SOLIMANDO, Mariella, Simone Schwarz-Bart dans la poétique du réel merveilleux. Essai sur

l'imaginaire antillais, Paris, L'Harmattan, 2008.

BERNABÉ, Jean, « Contribution à l'étude de la diglossie littéraire II. Le cas de Pluie et Vent sur Télumée Miracle », Textes et Documents, n² 2, Paris, Éditions Caribéennes, 1979.

BONNIOL, Jean-Luc, « Comment transmettre le souvenir de l'esclavage ? », Cités, $\mathrm{n}^{\circ} 1$, Presses Universitaires de France, 2006. 
CONDÉ, Maryse, « Autour d'une littérature antillaise », Présence Africaine, n 81, Paris, Présence Africaine, 1972/1.

CONDÉ, Maryse, Hérémakhonon, Paris, 10/18, 1976.

CONDÉ, Maryse, « Pluie et Vent sur Télumée Miracle, compte rendu de lecture », Présence Africaine, n 84 , Paris, $1972 / 2$

DUMONT, Jacques, L'amère patrie : histoire des Antilles françaises au XX $x^{e}$ siècle, Paris, Fayard, 2010.

DUPLAND, Edmond, Les Poètes de la Guadeloupe, Paris, Grassin, 1978.

GARCIN, Jérôme, « À la mémoire d'André Schwartz-Bart, le Blanc qui avait osé écrire sur les Antilles », Nouvel Observateur, Paris, article en ligne, 22 février 2015.

GYSSELS, Kathleen, «Le marranisme absolu dans l'œuvre d'André et de Simone Schwarz-Bart », Présence Francophone : André Schwarz-Bart et Simone Schwarz-Bart à Metz, n 79, Sherbrooke, 2012 GYSSELS, Kathleen, Marrane et marronne, la co-écriture réversible d'André et Simone Schwarz-Bart, Amsterdam, Rodopi, 2014.

IVOR-CASE, Frederick, The Crisis of Identity, Studies in the Guadeloupean and Martiniquan Novel, 1985. KAUFMANN Francine, « André Schwarz-Bart, le Juif de nulle-part », L’Arche, n 583, Paris, décembre 2006.

KAUFMANN, Francine, « Le projet judéo-noir d'André Schwarz-Bart : saga réversible », Présence Francophone : André Schwarz-Bart et Simone Schwarz-Bart à Metz, n 79, Sherbrooke, 2012.

KAUFMANN, Francine, « Les enjeux de la polémique autour du premier best-seller français de la littérature de la Shoah : Le Dernier des Justes, d'André Schwarz-Bart, prix Goncourt 1959 », Revue d'Histoire de la Shoah, $\mathrm{n}^{\circ}$ 176, septembre-décembre 2002.

LEVISALLES, Nathalie, « Quarante ans de Solitude », Paris, Libération, mai 2015.

ORBAN, Jean-Pierre, « Provocation du silence : André Schwarz-Bart », en ligne sur Diacritik.com, 24 juin 2020.

OUDIN BASTIDE, Caroline, « Fatalisme et aliénation : Pluie et Vent sur Télumée Miracle », CARE, Pointeà-Pitre, 1975.

PLougASTeL, Yann, « L'étoile du matin d'André Schwarz-Bart », Le Monde, Paris, 15 octobre 2009. SCHWARZ-BART, André et Simone, L'Ancêtre en Solitude, Paris, Seuil, 2015.

SCHWARZ-BART, André, « Pourquoi j'ai écrit La Mulâtresse Solitude », Paris, Le Figaro Littéraire, 1967. SCHWARZ-BART, André, dans « Simone et André Schwarz-Bart, sur les pas de Fanotte », propos recueillis par Roger et Héliane Toumson, Paris, T.E.D., 1979.

SCHWARZ-BART, André, Le Dernier des Justes, Paris, Seuil, 1959.

SCHWARZ-BART, André, lettre à Susan Dune, 1993, « Fonds Schwarz-Bart », Archives de la Bibliothèque nationale de France.

SCHWARZ-BART, Simone, Discours de réception du prix Carbet, Goyave, 2009.

SCHWARZ-BART, Simone, « Avant-propos », L'Ancêtre en Solitude, Paris, Seuil, 2015.

SCHWARZ-BART, Simone, au micro de Jacques Chancel, « Radioscopie : entretien avec Simone Schwarz-Bart », émission France-Inter du 4 octobre 1979. 
SCHWARZ-BART, Simone, Discours de réception du prix Carbet de la Caraïbe, Conseil Régional d'Ilede-France (Paris), 12 décembre 2008.

SCHWARZ-BART, Simone, et PloUgASTEL, Yann, Nous n'avons pas vu passer les jours, Paris, Grasset, 2019. SCHWARZ-BART, Simone, extrait du « Discours de réception du Prix des Lectrices de Elle » diffusé dans BARMA Claude, Ouvrez les guillemets, présentée par Bernard Pivot sur la $1^{\text {re }}$ chaîne de l'ORTF (Office de radiodiffusion-télévision française), émission du 21 mai 1973.

SCHWARZ-BART, Simone, Hommage à la femme noire (6 volumes), Bruxelles, Éditions Consulaires, 1988-1989.

SCHWARZ-BART, Simone, Pluie et Vent sur Télumée Miracle, Seuil, Paris, 1972.

TOUMSON, Roger, La Question d'identité, culture et dialectique de l'expérience en milieu antillais, actes du Colloque organisé par le Comité de la Culture, de l'Éducation et de l'Environnement du Conseil Régional de la Guadeloupe, Pointe-à-Pitre, 11-13 décembre 1989.

WENDLING, Rose-Marie, Église de Guadeloupe, nº 122, Pointe-à-Pitre, $2^{\mathrm{e}}$ quinzaine de septembre 1972.

\section{NOTES}

1. André et Simone SCHWARZ-BART, L'Ancêtre en Solitude, Paris, Seuil, 2015.

2. André SCHWARZ-BART, La Mulâtresse Solitude, Paris, Seuil, 1972.

3. Jérôme GARCIN, «À la mémoire d'André Schwartz-Bart, le Blanc qui avait osé écrire sur les Antilles », Nouvel Observateur, 22 février 2015, article en ligne sur bibliobs.nouvelobs.com.

4. Yann PlougAstel, "L'étoile du matin", d'André Schwarz-Bart ", Le Monde, 15 octobre 2009, article en ligne sur lemonde.fr.

5. Simone SCHWARZ-BART, Hommage à la femme noire (6 volumes), Bruxelles, Éditions Consulaires, 1988-1989.

6. Simone SCHWARZ-BART, Pluie et Vent sur Télumée Miracle, Paris, Seuil, 1972.

7. Jérôme GARCIN, « À la mémoire d'André Schwartz-Bart... », art. cit.

8. Les 26 et 27 mai 1967, la grève des ouvriers en bâtiment dégénère : la colère de milliers de personnes qui apprennent l'échec des discussions avec les délégués syndicaux au sujet de l'augmentation des salaires est réprimée dans le sang par les forces de l'ordre. On dénombre entre huit et plusieurs dizaines de morts parmi la population civile. Pour plus de détails, consulter le rapport Stora, remis le 21 novembre 2016 à la ministre des Outremers E. BAREIGTS et élaboré par la commission présidée par Benjamin STORA, composée des historiens Michelle ZANCARINI-FOURNEL, Jacques DUMONT, Serge MAM-LAM-FOUCK, Louis-Georges PLACIDE, Laurent JALABERT et Sylvain MARY. Consultable à l'adresse https://fr.calameo.com/read/000886379720261dabe09.

9. On retrouve des notes de lecture dans les journaux suivants imprimés en Guadeloupe : Clartés, 3 mars 1967 ; Les Alizés, n 4, avril-mai 1967; Madras, mai-juin 1967. Cette réception sera plus précisément explorée dans notre thèse, dirigée par D. Carlat et L. Cassin, Université Lyon 2 Université des Antilles.

10. De ce corpus ont été écartés les divers annuaires et bulletins, les documents administratifs ou les revues scientifiques portant sur des études précises de la faune et la flore des Antilles, comme «Fruits guadeloupéens et informations bananières" ou "Fondation pour la recherche au Surinam et aux Antilles néerlandaises ».

11. La revue Parallèle (1964-1971) se présente comme « une synthèse de l'activité littéraire et artistique des Antilles, diffusée en France, au Canada, au Sénégal »; à propos de cette revue, Dumont souligne qu'elle a pour but de «faire mieux connaître les Antilles, d'abord aux Antillais 
et leur donner éléments de savoir et réflexion et distance vis à vis du regard trop souvent valorisant porté de l'extérieur ».

12. La revue Acoma (1971-1973), dirigée par Édouard Glissant, se présente comme une revue de littérature, de sciences humaines et politiques aux Antilles Guyane et Caraïbes. L'ancrage historique, ethnographique et politique est prégnant. On peut citer à titre d'exemple quelques articles portés par cette revue : GLISSANT, GIRAUD et GAUDI, «Structures de groupes et tensions de groupes en Martinique », ou "Les conflits raciaux considérés comme substitut à la lutte des classes aux Antilles », ou "Évolution de l'économie sucrière en Martinique du XVII ${ }^{\mathrm{e}}$ au Xx ${ }^{\mathrm{e}}$ siècle », ou encore "Introduction à une étude des fondements socio-historiques du déséquilibre mental ", parus dans le premier numéro d'Acoma d'avril 1971. De même, GLISSANT, «Théâtre, conscience du peuple ", Acoma, n², juillet 1971, p.41-59. Elle est notamment le creuset où s'élaborent quelques-unes des théories glissantiennes.

13. Jacques DUMONT, «Identités et cultures, enjeux et mutations ", L'amère patrie, Paris, Fayard, 2010.

14. «Le CARE, pourquoi dire? ", CARE n 1, mars 1975, Pointe-à-Pitre, Guadeloupe, p. 5-9.

15. Voir Francine KAUFMANN, «Les enjeux de la polémique autour du premier best-seller français de la littérature de la Shoah : Le Dernier des Justes, d'André Schwarz-Bart, prix Goncourt 1959 ", Revue d'Histoire de la Shoah n 176, sept.-déc. 2002, Paris, Calmann-Lévy, p. 68-96 ; Simone schwARZBART et Yann PLOUGASTEL, Nous n'avons pas vu passer les jours, Paris, Grasset, 2019.

16. Hausses salariales, revalorisation du SMIG, équivalence de couverture sociale, dénonciation des effets pervers de l'immigration favorisée par le Bureau des migrations d'Outre-Mer (1963-1982). Voir notamment à ce sujet Jacques DUMONT, «Un univers en mutation: les années 60-70 ", L'amère patrie, op. cit.

17. Jean BENOÎT (dir.), L'Archipel inachevé. Culture et Société aux Antilles françaises, Presses de l'Université de Montréal ; et François GIROD, La Vie quotidienne de la société créole (Saint-Domingue au XVIII ${ }^{e}$ siècle), Paris, Hachette, 1972.

18. Cette revue (1967-1998) fonctionne comme un bulletin religieux du diocèse ; elle évoque la vie de l'Église locale et du diocèse et propose des articles de réflexion sur la Guadeloupe.

19. Rose-Marie WENDLING, Église de Guadeloupe, $\mathrm{n}^{\circ} 122$, $2^{\mathrm{e}}$ quinzaine de septembre 1972, p.4, périodique tiré à 700 exemplaires.

20. Celle-ci n'avait en effet pas apprécié « ce roman, premier bestseller de la littérature de fiction consacrée à la Shoah, [...] fondé sur l'histoire de l'antisémitisme chrétien depuis les Croisades jusqu'à la Shoah incluse », résumé emprunté à Francine KAUFMANN, «Le projet judéo-noir d'André Schwarz-Bart : saga réversible », Présence Francophone : André Schwarz-Bart et Simone Schwarz-Bart à Metz, $\mathrm{n}^{\circ}$ 79, Sherbrooke, 2012.

21. Tout comme le soulignait l'inscription dans Guadeloupe 2000: faut-il en effet rappeler qu'Édouard Boulogne, personnalité encore très décriée, notamment en Guadeloupe, est issu d'une longue lignée de Blancs créoles, se définit lui-même comme « français d'origine créole et de sensibilité chrétienne». Ses détracteurs le considèrent comme un chrétien proche des intégristes, qui partagent une vision complaisante de l'esclavage.

22. Les journalistes qui rédigent des critiques littéraires dans les journaux et revues que nous avons pu consulter choisissent en effet de mettre à l'honneur des écrits engagés, révoltés, centrés sur la situation politique et sociale de la Guadeloupe, comme ceux d'auteurs comme Sonny Rupaire, dont le recueil bilingue français-créole Cette igname brisée qu'est ma terre natale paraît en 1971. On suppose ici qu'ils s'adaptent aux centres d'intérêt de leurs lecteurs.

23. «[...] Solitude échappe à son auteur, dont la paternité est reniée et à qui certains dénient le droit de la revendiquer », Francine KAUFMANN, « Le projet judéo-noir d'André Schwarz-Bart : saga réversible » Présence francophone: André Schwarz-Bart et Simone Schwarz-Bart à Metz, $\mathrm{n}^{\circ} 79$, Paris, 2012, p. 15-38. 
24. Nous écartons de notre étude des récits de témoins indirects, comme celui d'Ernest Pépin que l'on retrouve sous forme romanesque dans La Souvenance, CaraibEditions, 2020, p. 80 (chapitre qui prend la forme d'une lettre adressée à André Schwarz-Bart par Simone Schwarz-Bart), et dans un entretien accordé à Francine Kaufmann en 2012 (présent dans ce numéro).

25. Simone SCHWARZ-BART, discours de réception du prix Carbet, Goyave, 2009.

26. Simone SCHWARZ-BART, extrait du discours de réception du prix des Lectrices de Elle diffusé dans Claude BARMA, Ouvrez les guillemets, présentée par Bernard Pivot sur la $1^{\text {re }}$ chaîne de l'ORTF (Office de radiodiffusion-télévision française), émission du 21 mai 1973.

27. Et donc avant la médiatisation dans les milieux parisiens qui suit immédiatement l'annonce de la remise du prix des lectrices de Elle, soit après mai 1973.

28. Après 1968, les facultés deviennent des « Unités d'Étude et de Recherche ", puis des Unités de Formation et de Recherche. Dès 1972, ces Unités deviennent Centres Universitaires.

29. Le passage coupé est reporté et analysé un peu plus loin dans cette étude.

30. Anonyme, La Vérité, n 76, février 1973, p. 4, numéro tiré à 4000 exemplaires. La revue parait de 1967 à 1975.

31. Ibid.

32. Jacques DUMONT, L'amère patrie..., op. cit.

33. Et notamment ceux qui formeront le futur comité de lecture de la revue CARE. Cette revue, celle du Centre Antillais de Recherche et d'Études, est créée en mars 1975 par Harry Boucard contre ce qui est décrit comme un « cannibalisme du discours " grevant le débat intellectuel aux Antilles. Voir à ce propos l'éditorial du deuxième numéro de la revue : CARE, n² 2, juin 1975.

34. Ils vivent alors entre Lausanne et Paris.

35. Harry BOUCARD, entretien téléphonique de mars 2019, mené dans le cadre de notre travail de thèse.

36. Edmond DuPLAND, Les Poètes de la Guadeloupe, Paris, Grassin, 1978, p. 10.

37. Anonyme, La Vérité, op. cit.

38. Simone SCHWARZ-BART, extrait du discours de réception du prix des Lectrices de Elle, op. cit.

39. Au cours de l'année 2019-2020, nous avons eu la chance de pouvoir échanger avec les professeurs de l'époque Harry Boucard et Jacky Dahomay, et des étudiants présents à la conférence, Michel Hippon, Renée Reynaud et Jean-Pierre Sainton.

40. Fritz Gracchus est alors professeur de philosophie à l'École normale. Sa thèse de doctorat, Les lieux de la mère dans les sociétés afroaméricaines. Pour une généalogie du concept de matrifocalité, soutenue à l'Université de Paris VII en 1978 et publiée par les Éditions Caribéennes/CARE en 1980, préfacée par le professeur Jacques André qui a aussi enseigné en Guadeloupe, réinterroge le fonctionnement et la structure de la composition familiale aux Antilles, et notamment les concepts de « matrifocalité » et de « matrilinéarité ».

41. Edmond DUPLAND, op. cit., p. 10.

42. Quoique tous s'accordent sur la réputation d'un Fritz Gracchus à l'esprit frondeur, qui n'aurait pas non plus hésité à qualifier publiquement la conférence de Jack Corzani en 1971 d'« échec».

43. Jean BERNABE, «Contribution à l'étude de la diglossie littéraire II. Le cas de Pluie et Vent sur Télumée Miracle, Textes et Documents, n² 2, Paris, Éditions Caribéennes, 1979, p. 103.

44. Nathalie LEVISAllES, citant Simone Schwarz-Bart, dans "Quarante ans de "solitude" ", Libération, 27 mai 2015.

45. André SCHWARZ-BART, Le Dernier des Justes, Paris, Seuil, 1959.

46. Cette piste est plus largement et longuement explorée dans notre travail de thèse.

47. Nathalie LEVISALLES, op. cit.

48. Lettre d'André Schwarz-Bart à Paul Flamand datée du 5 décembre 1972, «Dossier SchwarzBart », Archives du Seuil sauvegardées à l'IMEC sous la côte SEL 392.1.1. 
49. La langue est d'abord un outil politique, et de nombreuses réunions dans les cercles syndicaux et autonomistes se font en créole. Le GEREC-F (Groupe d'Études et de Recherches en Espace Créolophone et Francophone), qui se consacre à l'étude et à l'apprentissage des créoles francophones et au développement du créole écrit, n'est créé par Jean Bernabé et Donald ColatJolivière qu'en 1975.

50. Michel HIPPON, entretien réalisé le 14 février 2020.

51. Jean-Pierre SAINTON, entretien téléphonique du juin 2019.

52. Renée REYNAUD, entretien du 8 février 2020.

53. Simone schWARZ-BART et Yann PloUgASTel, Nous n'avons pas vu passer les jours, Paris, Grasset, 2019, p. 146-156.

54. Kathleen GYSSELS, Marrane et marronne. La co-écriture réversible d'André et de Simone Schwarz-Bart, Amsterdam, Rodopi, coll. « Faux Titre », 2015, p. 401.

55. Si nous nous en tenons (faute de preuve plus tangible) notamment à l'hypothèse selon laquelle l'article de La Vérité serait un reflet des opinions du "peuple " guadeloupéen, par opposition à celles des milieux intellectuels et bourgeois.

56. André SCHWARZ-BART, cité dans Simone SCHWARZ-BART et Yann PLOUGASTEL, op. cit.

57. Comme le recueil de Sonny Rupaire qui circule d'abord, comme le ferait un pamphlet politique, essentiellement dans les milieux indépendantistes.

58. Simone SCHWARZ-BART, « Avant-propos », L'Ancêtre en Solitude, Paris, Seuil, 2015, p. 12-13.

59. Jérôme GARCIN, op. cit.

60. Voir notamment Kathleen GYSSELS, "Le marranisme absolu dans l'œuvre d'André et de Simone Schwarz-Bart", Présence Francophone: André Schwarz-Bart et Simone Schwarz-Bart à Metz, $\mathrm{n}^{\circ}$ 79, 2012 ; et Marrane et marronne, la co-écriture réversible d'André et Simone Schwarz-Bart, Amsterdam, Rodopi, 2014.

61. Maryse CONDÉ, « Pluie et Vent sur Télumée Miracle, compte rendu de lecture », Présence Africaine, $\mathrm{n}^{\circ} 84,1972 / 2$, Paris, p. 138-139.

62. Sur ce sujet, voir notamment Xavier LUCE et Fanny MARGRAS, «Schwarz-Bart et Condé... du pays natal retrouver le fumet: Relecture critique de la recension de Pluie et Vent sur Télumée Miracle (Simone Schwarz-Bart, 1972) par Maryse Condé », dans ce même numéro de Continents Manuscrits.

63. Maryse CONDÉ, op. cit, p. 138.

64. Maryse CONDÉ, op. cit, p. 139.

65. Maryse CONDÉ, « Autour d'une littérature antillaise », Présence Africaine, $n^{\circ} 81$, Paris, Présence Africaine, 1972/1, p. 170-176; au sujet de Jack Corzani, Encyclopédie antillaise. Littérature antillaise (Poésie), Paris, Éditions Desormeaux, 1971, 318 p.

66. Maryse CONDÉ, Hérémakhonon, Paris, 10/18, 1976.

67. Roger toumson, La Question d'identité, culture et dialectique de l'expérience en milieu antillais, actes du Colloque organisé par le comité de la Culture, de l'Éducation et de l'Environnement du Conseil régional de la Guadeloupe, Pointe-à-Pitre, 11-13 décembre 1989.

68. Jacques DUMONT, op .cit.

69. Ainsi, par exemple, au CESL, Émile Yoyo, missionné depuis la Martinique, travaille sur les contes et les romans haïtiens; il remplace Jack Corzani qui entre 1965 et 1971 proposait une « découverte des littératures négro-africaine » à travers les œuvres de Léopold Sédar-Senghor, Léon Gontran-Damas et Aimé Césaire notamment, à l'Université. Un peu plus tard, Jacques André, arrivé en Guadeloupe au titre de la coopération, enseigne la littérature antillaise à l'université, puis devient professeur de psychologie à l'École normale de Pointe-à-Pitre.

70. Jacques ANDRÉ, « Parcours libidinal d'une œuvre : La Vie et la Mort de Marcel Gonstran de Vincent Placoly ", CARE, numéro spécial, Pointe-à-Pitre, 1976. Il importe de noter que ce numéro débute sur des réflexions précises sur la manière dont on pourrait définir la littérature antillaise : «Si les 
mots demeurent ceux de l'Autre [...] leur combinaison, elle, devra renvoyer au lecteur l'image d'une authenticité retrouvée ", p. 5.

71. Maryse CONDÉ, op. cit, p. 139.

72. Caroline OUDIN-BASTIDE, "Fatalisme et aliénation: Pluie et Vent sur Télumée Miracle», CARE, Pointe-à-Pitre, 1975.

73. Simone SCHWARZ-BART, au micro de Jacques CHANCEL, "Radioscopie : entretien avec Simone Schwarz-Bart », France-Inter, 4 octobre 1979.

74. Francine KAUfMANN, «André Schwarz-Bart, le Juif de nulle-part», L'Arche, n 583, Paris, décembre 2006, p. 84-89.

75. Jean-Luc BonNiol, «Comment transmettre le souvenir de l'esclavage? », Cités, $\mathrm{n}^{\circ} 25$, PUF, 2006/1, p. 181-185.

76. André SCHWARZ-BART, lettre à Susan DUNE, 1993, «Fonds Schwarz-Bart», Archives de la Bibliothèque nationale de France.

77. Thomas R. EDWARDS, THE NYRB, 22 mars 1973, p. 30-31, cité dans Kathleen GYSSELS, Marrane et marronne, la co-écriture réversible d'André et Simone Schwarz-Bart, Amsterdam, Rodopi, 2014.

78. Frederick IVOR CASE, The Crisis of Identity: Studies in the Guadeloupean and Martiniquan Novel, Sherbrooke, Naaman, 1985.

79. Kathleen GYSSELS, Marrane et marronne..., op. cit., p. 82-83.

80. Frederick de IVOR CASE, The Crisis of Identity..., op. cit.

81. Simone SCHWARZ-BART et Yann PLOUGASTEL, op. cit. p. 159-160.

82. Nous reprenons ici le terme de Jérôme Garcin.

83. Voir à ce sujet Jean-Pierre ORBAN, « Provocation du silence : André Schwarz-Bart », Diacritik, 24 juin 2020, en ligne sur diacritik.com.

84. Francine KAUFMANN, op. cit.

85. Francine KAUFMANN, "André Schwarz-Bart, le Juif de nulle part ", L’Arche, ${ }^{\circ} 583$, décembre 2006, p. 84-89

86. Simone SCHWARZ-BART et Yann PLOUGASTEL, op. cit. p. 145.

87. André schWARZ-BART, "Pourquoi j'ai écrit LA MULÂTRESSE SOLITUDE », Paris, Le Figaro littéraire, 1967.

88. Simone SCHWARZ-BART et Yann PloUgASTEL, op. cit., p. 146-156.

89. Daniel MAXIMIN, entretien téléphonique du 24 janvier 2020.

90. Syto Cavé signe la première mise en scène de Ton Beau Capitaine à Pointe-à-Pitre le 28 avril 1987, dans une production de Michèle Montantin.

91. André SCHWARZ-BART, dans «Simone et André Schwarz-Bart, sur les pas de Fanotte», propos recueillis par Roger et Héliane Toumson, Paris, T.E.D., 1979, p. 14.

92. Voir à ce sujet l'article de Jean-Pierre ORBAN, « Provocation du silence : André Schwarz-Bart », art. cit. et consulté le 24 juin 2020.

93. C'est dans l'espoir de compléter ces éléments de réponse que nous menons notre travail de recherche sur la chronologie de la réception des œuvres des Schwarz-Bart.

94. Simone SCHWARZ-BART, discours de réception du prix Carbet de la Caraïbe, Conseil régional d'Ile-de-France, 12 décembre 2008, en ligne sur dailymotion.com (« Conférences ITM (M. GLISSANT - G. ROBILLARD) ». 


\section{RÉSUMÉS}

La réception du dernier roman signé par André Schwarz-Bart de son vivant en Guadeloupe est souvent présentée comme houleuse, donnant lieu à un parallèle entre les attaques émises par la communauté juive à l'encontre du Dernier des Justes en 1959 et celles qui auraient été formulées par la communauté antillaise contre La Mulâtresse Solitude en 1972. Nous proposons ici un parcours historique et littéraire de la réception antillaise des œuvres des Schwarz-Bart en 1972 : celui-ci nous permet d'éclairer un pan de l'histoire du couple d'auteurs.

\section{INDEX}

Mots-clés : André Schwarz-Bart, Simone Schwarz-Bart, réception des œuvres, littérature antillaise, La Mulâtresse Solitude, Pluie et vent sur Télumée Miracle, légitimité d'écriture, silence littéraire

\section{AUTEUR}

\section{FANNY MARGRAS}

FANNY MARGRAS bi-admissible à l'agrégation de Lettres modernes, est actuellement doctorante contractuelle sous la direction des professeurs D. Carlat (Université Lumière Lyon 2), R. Toumson (Université des Antilles) et de Mme L. Carvigan-Cassin (Université des Antilles). Chercheuseassociée à l'ITEM-CNRS, membre de l'association CARACOL (Observatoire des littératures caribéennes) et de l'équipe de recherche Schwarz-Bart de l'ITEM, elle consacre ses travaux de recherche à la collaboration littéraire entre André et Simone Schwarz-Bart. Elle est l'auteure de « Les corps de Solitude : exploration des adaptations théâtrales du roman schwarz-bartien » (colloque « Adaptations du texte littéraire américano-caraïbe : formes et enjeux », du 20-22 novembre 2019) et de l'article « Adieu Bogota : naissance d'un écrit bifrons », à paraître dans Nouvelles Études francophones en 2021. 